\title{
Publisher Correction: Tau folds differently between diseases
}

\section{Ian Fyfe}

Nature Reviews Neurology (2018) https://doi.org/10.1038/s41582-018-0076-x

Published online 19 September 2018

In the original version of this article published online, Michel Goedert's first name was spelt incorrectly and the affiliation of Sjors Scheres was incorrect. These errors have been corrected in the HTML and PDF versions of the article.

https://doi.org/10.1038/s41582-018-0089-5 I Published online 9 October 2018 